\title{
Therapeutic Contract and Ethical Practice in Counselling and Psychotherapy
}

\author{
Sunjida Shahriah ${ }^{1}$, Sunjida Islam ${ }^{2}$, Khalid Arafat ${ }^{3}$ \\ 1. Professor \& Director, Phoenix Wellness Centre, Dhaka, Bangladesh. \\ Email:sshahriah@yahoo.com (corresponding author) \\ 2.. Counsellor and Psychologist, Phoenix Wellness Centre, Dhaka, Bangladesh. \\ 3. Coordinator, Phoenix Wellness Centre, Dhaka, Bangladesh.
}

\begin{abstract}
Psychotherapists and counsellors confront several ethical dilemmas as they tend to provide effective services. There has been much debate among psychotherapists and counsellors alike around the utility of therapeutic contracts. Some view contracts as being restrictive to the therapeutic process and often hindering the work done in sessions. In contrast, many counsellors and psychotherapists use those agreements to revisit specific therapeutic topics and establish the guidelines necessary for this professional arrangement. No matter the opinion or preference of contracts, the development of written and/or verbal consent of specific topics in psychotherapy remains essential. This remains one of the formal features of the arrangement and starting relationship in current counselling and psychotherapy practice. This paper aims to discuss the necessity and ethical demand of therapeutic contract in counselling and psychotherapy practice.
\end{abstract}

Key words: Therapeutic contract, counselling, psychotherapy, ethics.

Introduction: Therapeutic contract is a mutual agreement in counselling or psychotherapy, among the therapist and the client. It indicates the rights and responsibilities of both to ensure target treatment goal. Contracting also ensures that "the counseling process will be performed in a good and safe manner and, as a written document, provides the necessary space for legal intervention if the responsibilities outlined are not met"1. Specific contract is mandatory to assess the output of individual session to ensuring both ethical concerns and the therapeutic process. A practitioner evaluates the situation and context of a contract for better management of the client. The practitioner and client are related to each other as equals. Hence, they share responsibility for the change the client wants to make ${ }^{2}$. There are already considerable discussions and debate on the utility of contracts among psychotherapists and counsellors alike. Some see contracts as restricting the healing process and often hindering the work conducted in sessions. The agreement can restrict both the capacity to perform specific treatment procedures and the scope of the role of the therapist. The innovation and ability for clients to extend their limits of freedom could be undermined by defined protocols or contracts ${ }^{3}$. On the contrary, those arrangements are used by certain therapists to revisit specific individual therapy subjects and create the appropriate criteria for this professional structure $^{3}$. Contracts create an equilibrium of power and balance for sessions very often ${ }^{4}$. No matter the view or desire of contracts, it remains important to develop written and/or verbal agreement for a particular 
psychotherapy subject/patient. This part is one of the official aspects of the psychotherapy agreement and original partnership, whether in private practice, community clinics, hospitals, schools, or in the legal system ${ }^{3}$. This paper aims to discuss the necessity and ethical demand of therapeutic contract in counselling and psychotherapy practice.

Theoretical Concepts: Psychotherapists differ widely in the utility of contracts in practice, beyond the theoretical origins of psychotherapy contracts ${ }^{3}$. Traditionally from the psychoanalytic field, the first contracts used formally in therapy emerged, where the arrangement was used mostly like a one-way negotiation with the client's desires that the therapist had. In Freudian perspectives, both the client and the therapist are allowed to break a contract at any point, as this "separation was not seen as a negative reaction, but rather a choice to leave certain problems unresolved"4. Family structures and ecological constructs were the first to incorporate contracts as an integral method of boundary setting, as the utility of a therapeutic contract grew over time. Contracts not only offered protections between other family members but from individuals involved in the treatment process outside of therapy ${ }^{5}$. In the 1960s, during the development of brief therapy approaches and treatments, therapeutic contracting became more prevalent in psychotherapy ${ }^{6}$. The use of contracts as a critical aspect of treatment has been highlighted by short-term models, as described by Tudor ${ }^{7}$. The method that therapy can occur over time has always been determined by contracts that are organized and precise in course of care and nature. It is especially applicable for those who are placed in residential treatment, hospital or short-term rehabilitation settings and have specific contracts to address certain medical or mental health issues ${ }^{7}$. However, more recently, psychotherapists and counsellor from the postmodern perspective see therapeutic contracts as a socially constructed process, as they came into the therapeutic agreement with preconceived notions about what the contract looked like or what the expectations for therapy entailed, along with the goals for therapy most often phrased in the "language of the clients, where more strengths, solutions, and narratives are emphasized in the initial contract"'

Therapeutic Contract and Ethics: It is not surprising that the intentions and usage of contracts in sessions vary among counsellors and psychotherapists. Typically, counselling contracts begin with a short overview of the services that are offered to the clients, as highlighting the treatment specifications delivered by the professionals ${ }^{8}$. Commonly, psychotherapists are seen as facilitators with basic therapeutic objectives, expected to focus with during the process of mental health care for individuals. However, in the therapeutic process, clients sometimes may feel unnoticed or unheard, jeopardizing their desire to address broader problems beyond the expectations agreed upon ${ }^{3}$.

Historically, psychotherapists have used three forms of therapeutic contracts. Firstly, an administrative contract which deals with therapy's logistical supports, such as location, venue, length, details of the organization, and secrecy. To highlight the 
setup and configurations of the counseling process, this is regarded as a fundamental text $^{9}$. Secondly, a written contract, the document that the therapist has formulated for a particular cause or intent that helps to describe treatment. This establishes trust and confidence that the therapist will work in psychotherapy/counselling on a particular topic or that in meetings the therapist will honour a clear ethical/legal issue ${ }^{9}$. Finally, the purpose and focus of therapy is established by a professional contract. On what the emphasis of the job will be, the therapist and client share a legally binding arrangement. Here collaborative and mutually agreed attempts are taken to set targets ${ }^{9}$.

There are some ethical facets of a traditional contract with counsellor/psychotherapist that should be addressed in therapeutic contract: ${ }^{3}$

1) The treatment type/style that the counsellor/psychotherapist is offering;

2) The types of resources given and what exists in a normal session;

3) How long treatment will take and over what specified time frame;

4) Payment procedure/arrangements and how postponed appointments are dealt with;

5) How to discuss questions of secrecy and their boundaries in counselling and psychotherapy;

6) How to deal with unforeseen events during treatment.

The relationships between the client and the counsellor/psychotherapist also more subjective and less formalized, as goals are typically co-constructed and chosen, and counsellor/psychotherapist works as facilitator providing fresh insights and visions on what objectives are to be accomplished. However, one of the disadvantages of such contracts is that in times of distress or turmoil, it also does not arrange the lives of clients and families efficiently. Moreover, to decide what progress happens in the counselling phase and when cessation of care is implemented, the therapist can experience some uncertainty ${ }^{1,3,10}$. The body of a contract should start with confidentiality statements, positive words and continue to the specifics of the meetings, how the therapy will commence, how long it will last, how it will be completed, facts about missing meetings, where and how the sessions will take place. Information on reimbursement, rates, and how various conditions impact these rates (e.g. missed visits, forms of psychotherapy delivered) should be concluded in contracts $^{3,9,10}$. In order to outline the rules and steps for mandatory documentation and other legal responsibilities, counsellor and psychotherapists should be motivated enough to state the ethical code(s) of their assigned occupation or professional code of ethics as followed by the corresponding national association ${ }^{11,12}$.

For the safety and ethical clearance, appropriate informed consent from clients about the process, procedures, plan, and risks vs. benefits of the treatment is mandatory $^{3,11,12}$. All professional counsellors and psychotherapists must also get written consent about any videotaping, audio recording, or permitting third-party observation taking place ${ }^{11,12}$. Moreover, for authorizations to release or obtain information to outside parties, the they must 
obtain consent from each individual competent to execute this waiver. Verbal contracting is usually not enough consent for in the ethical codes ${ }^{11,12}$. From the ethical point of view, the therapeutic contract should address how information will be either shared with or kept confidential from other members of the system. Data from cannot be published without permission from client involved in therapy $y^{3,11,12}$. For some families, there may be good reasons for information to be kept confidential (e.g., safety for a partner in a violent environment, establishing rapport with an adolescent in a family system $)^{3,9,13}$. Besides, other therapeutic systems may benefit from a "no secrets" policy within the contract to protect the best interest of the system (the identified patient in a systemic approach) from information that might be in conflict with therapeutic goals ${ }^{3,13}$. With a "no secrets" policy stated directly in the contract, the therapist is able to exercise his or her clinical judgment regarding the need to share privately disclosed information with the system. While information may be ethically shared with other members in the therapy process, any communication with third parties (e.g., individual therapists, physicians, schools, attorneys, and other family members outside the therapeutic system) requires an authorization to release information. Information from a couple's or family's treatment may not be released without permission from each person involved in therapy, while for minors, parental permission should be mandatory $^{13,14}$. Conversely, in case of minors, besides parental consent and contract, an assent should be taken from the minor patient to make it ethically sound ${ }^{14,15}$. There have been debates in the mental health field regarding the use of "no-harm contracts" for decades. These contracts are sometimes used when a partner or family member struggles with suicidal ideation or other self-harming behaviors, like cutting or over-medicating $^{16}$. A variety of reported risks have been advocated against the use of no-harm or no-suicide arrangements with depressed clients ${ }^{3,16}$. Some clients may feel a sense of faith in their protection, knowing that the contract protects the therapist rather than the client. Others could withhold information about a recent suicide attempt or actions, fearing that disclosure of this information may jeopardize their therapy status ${ }^{16}$. Alternatingly, many have also used safety planning as a way to recognize early risks of self-harm, protect the home environment, and use other services. A "nosubstance use contract" can facilitate productive explorations of the addictive behaviors of individuals. Allowing the substance abuser to identify the parameters of this contract (e.g., abstinence vs. limited use of the substance, length of time) places responsibility on that family member and, in many cases, can help him or her acknowledge addictive behaviours ${ }^{17}$. It may imply in any ethical or self-harm issues such as drug abuse, pornographic addiction, gambling, practicing unsafe sex, cyber bullying threat, sexual harassment, etc. Such as emergency medical or legal support need must be notified immediately to a safe person declared by the client earlier ${ }^{16,17}$.

Conclusion: In professional practice, both the counsellor/ psychotherapist and the client should integrate concrete expectations 
into the treatment contract with an awareness of the problem. The therapist and each member of the system should collaboratively agree on goals. It will be important for the counseling system to determine how many sessions will occur. There must be a clear agreement how both client and therapist will understand the measurable and observable change that desired treatment contract has been fulfilled. The therapist is free to exercise his or her professional maturity on the need of the patients, to act on the best interest of the patients and adopt policies specified explicitly in the contract on possible ethical issues that may arise during sessions.

\section{References:}

1. Beahrs JO, Gutheil TG. Informed consent in psychotherapy. Am J Psychiatr. 2001;158(1):410.

2. Stewart I, Joines V. TA Today: a new introduction to transactional analysis. Nottingham: Life space Publishing; 1987.

3. Zubatsk M, Hiefner A. Therapeutic contract in couple and family therapy. In: Lebow J, Chambers A, Breunlin D. (eds). Encyclopedia of couple and family therapy. Philadelphia: Springer; 2017.

4. Sills C. Contracts in counseling and psychotherapy. Newbury Park: Pine Forge Press; 2006.

5. Gray A. An introduction to the therapeutic frame. New York: Routledge; 2013.

6. Elton-Wilson J. Integration and eclecticism in brief time-focused therapy. In: Palmer S, Woolfe R. (eds.). Integrative and eclectic counselling and psychotherapy. London: Sage Publication; 1999.

7. Tudor K. (ed.). Transactional analysis approaches to brief therapy: what do you say between saying hello and goodbye? Thousand Oaks: Sage; 2002.
8. Beall L. The corrupt contract: problems in conjoint therapy with parents and children. Am J Orthopsychiatr. 1972;42(1):77-81.

9. Croarkin P, Berg J, Spira J. Informed consent for psychotherapy: a look at therapists' understanding, opinions, and practices. Am J Psychother. 2003;57(3):384-400.

10. Barnett JE. The ethical practice of psychotherapy: easily within our reach. J Clin Psychol. 2008;64(5):569-575.

11. Canadian Counselling and Psychotherapy Association (CCPA). The Code of Ethics. 2017. (Available at: https:/www.ccpa-accp.ca/wpcontent/uploads/2014/10/CodeofEthics_en.pdf). (Accessed on February 14, 2019).

12. American Counseling Association (ACA). ACA Code of Ethics. 2014. (Available at: https://www.counseling.org/docs/defaultsource/default-document-library/2014-code-ofethicsfinaladdressc97d33f16116603abcacff0000bee5e 7.pdf) (Accessed on February 15, 2019).

13. Lebow J. Integrative family therapy for disputes involving child custody and visitation. J Fam Psychol. 2003;17(2):181-192.

14. Redding RE. Children's competence to provide informed consent for mental health treatment. Wash Lee Law Rev. 1993;50(2):695-753.

15. McCabe MA. Involving children and adolescents in medical decision making: developmental and clinical considerations. J Pediatr Psychol. 1996;21(4):505-516.

16. Farrow TL, O'Brien AJ. 'No-suicide contracts' and informed consent: an analysis of ethical issues. Nurs Ethics. 2003;10(2):199-207.

17. Diamond J. Narrative means to sober ends: treating addiction and its aftermath. New York: Guilford; 2000.

Authors' contribution: S Shahriah was involved in concept and design; S Shahriah, S Islam, K Arafat were equally involved in the literature search, compilation, manuscript writing and revision.

Conflict of interest: None declared. 